\title{
Neighboring Extremal Controllers for Singular Problems
}

\author{
S. Gros, B. Srinivasan, D. Bonvin \\ Laboratoire d'Automatique \\ Ecole Polytechnique Fédérale de Lausanne \\ CH-1015 Lausanne
}

September 29, 2003

\begin{abstract}
:
The dynamic optimization problem in the presence of uncertainty (model mismatch and disturbances) is addressed. It has been recently proposed that this problem can be solved by tracking the necessary conditions of optimality in the various intervals of the solution. In this paper, it is shown that the standard neighboring extremal approach, which uses linearization around the optimal trajectory, drives to zero the first-order variation of the necessary conditions of optimality on the parts of the solution where no constraint is active. This fact is used to extend the neighboring extremal approach to singular problems. In this last case, the nonlinear dynamic provides the information lacking in the first-order approximation, hence both the linearization and the nonlinear dynamics are used.
\end{abstract}

Keywords: Dynamic optimization, Optimal control, Measurement-based optimization, NCO-tracking, Neighboring extremals, Singular problems. 


\section{Introduction}

Dynamic optimization provides an unified framework for improving process operations while taking into account operational and other types of constraints $[1,7]$. Recently, there has been some emphasis on using measurements in the optimization framework in order to handle the uncertainty (model mismatch, process variations and disturbances) that is inevitably present in a real process. Among the various measurement-based optimization methods available in the literature $[4,6]$, a promising one, labeled NCOtracking, consists of enforcing the Necessary Conditions of Optimality corresponding to the uncertain situation [6].

NCO-tracking uses the fact that there are only two types of arcs that can constitute the optimal solution: constraint-seeking and sensitivity-seeking arcs [7]. This distinction depends on whether the solution is determined by the constraints of the optimization problem or forces a sensitivity (gradient) to zero. When the solution is determined by the constraints, tracking the necessary conditions of optimality corresponds to enforcing the corresponding constraints. The other case of sensitivity-seeking arcs, which is typically more involved, will be considered in this paper.

Along a sensitivity-seeking arc, optimization can be treated as the regulation of a sensitivity around zero. The main difficulty arises from the fact that this gradient information depends on the adjoint variables that are typically unknown. Thus, an efficient way of estimating them and their variations is necessary. A simple technique that has been used for over four decades is the sweep method where, for a linear (linearized) system, the adjoint variables (or their variations) are considered proportional to the state variables (or their variations). The linearized version of this approach leads to the Neighboring Extremal (NE) controller [2,3,5] which is revisited in this paper. It is shown that the standard NE controller forces to zero the first-order variation of the NCO. Thus, the NE controller can in fact be used for NCO-tracking.

The main contribution of this paper lies in the use of the link between NE controllers and NCO-tracking to extend the NE controllers to the singular case. The singular case arises when the optimal inputs cannot be computed directly from the NCO, and time differentiations of the $\mathrm{NCO}$ are required. In such a case, the standard NE controller cannot be used since it calls for inversion of a singular matrix. Time differentiations of the first-order variation of the $\mathrm{NCO}$ are used to derive the $\mathrm{NE}$ controller in the singular case. 
The paper is organized as follows. In Section 2, the necessary conditions of optimality are derived and the standard neighboring extremal controller is presented. Also, a link between the two is established. In Section 3, the neighboring extremal approach is extended to singular systems. An application example is presented in Section 4, and Section 5 concludes the paper.

\section{Neighboring Extremal Approach for Non- singular Problems}

\subsection{Dynamic optimization}

Consider the following dynamic optimization

$$
\begin{aligned}
u^{*}(t) & =\arg \min _{u(t)} J \\
\text { s.t. } \quad J & =\Phi\left(x\left(t_{f}\right)\right)+\int_{0}^{t_{f}} L(x, u) d \tau \\
\dot{x} & =F(x, u) \quad x(0)=x_{0} \\
S(x, u) & \leq 0, \quad T\left(x\left(t_{f}\right)\right) \leq 0
\end{aligned}
$$

where $x$ corresponds to the states of the system, $u$ the inputs, $F$ the system dynamics, $J$ the scalar objective function to be minimized, $t_{f}$ the fixed final time, $\Phi$ the terminal cost, $L$ the integral cost, $S$ the path constraints, and $T$ the terminal constraints. The solution of this problem is typically discontinuous and consists of several intervals with corresponding arcs. Along the various arcs, the solution is either (i) determined by the constraints of the optimization problem, or (ii) inside the feasible region. Only the latter case will be considered here and, thus, the constraints $S$ and $T$ will not be considered.

The notation $a_{b}=\frac{\partial a}{\partial b}$ will be used. When the solution is inside the feasible region, i.e. when no constraints are active, the Necessary Conditions of Optimality (NCO) can be expressed as:

$$
H_{u}=\lambda^{T} F_{u}+L_{u}=0
$$

where the Hamiltonian is given by $H=\lambda^{T} F+L$, with the adjoints $\lambda$ governed by the following equations:

$$
\dot{\lambda}=-H_{x}^{T}, \quad \lambda\left(t_{f}\right)=\Phi_{x}\left(t_{f}\right)
$$


Since along the arcs where none of the constraints are active, the solution seeks to push the sensitivity $H_{u}$ to zero, such an arc will be referred to as a sensitivity-seeking arc.

\subsection{Neighbouring Extremal Approach}

In the presence of the perturbations $\delta x$ and $\delta u$ around the optimal trajectories, the cost becomes as $J=J_{\text {nom }}+\delta J+\delta^{2} J+O\left(\delta^{3} J\right)$, where $J_{\text {nom }}$ is the nominal optimal cost, and $\delta J$ and $\delta^{2} J$ are given by [3]

$$
\begin{gathered}
\delta J=\Phi_{x} \delta x\left(t_{f}\right)+\int_{0}^{t_{f}}\left(H_{x} \delta x+H_{u} \delta u\right) d \tau \\
\delta^{2} J=\frac{1}{2} \delta x\left(t_{f}\right)^{T} \Phi_{x x} \delta x\left(t_{f}\right)+\frac{1}{2} \int_{0}^{t_{f}}\left[\begin{array}{ll}
\delta x^{T} & \delta u^{T}
\end{array}\right]\left[\begin{array}{cc}
H_{x x} & H_{x u} \\
H_{u x} & H_{u u}
\end{array}\right]\left[\begin{array}{c}
\delta x \\
\delta u
\end{array}\right] d \tau
\end{gathered}
$$

For an extremum, $\delta J=0$ [3]. Ignoring the terms of order 3 and higher, the neighboring extremal (NE) approach minimizes $\delta^{2} J$ subject to the linearized system equations:

$$
\begin{aligned}
\delta u^{*}(t) & =\arg \min _{\delta u(t)} \delta^{2} J \\
\text { s.t. } \quad \dot{\delta x} & =F_{x} \delta x+F_{u} \delta u
\end{aligned}
$$

For this NE problem with the state $\delta x$, the inputs $\delta u$ and the adjoints $\bar{\lambda}$, the corresponding Hamiltonian, $\bar{H}$, the adjoint equations and the necessary condition of optimality are given by

$$
\begin{aligned}
& \bar{H}=\bar{\lambda}^{T}\left(F_{x} \delta x+F_{u} \delta u\right)+\frac{1}{2}\left[\begin{array}{ll}
\delta x^{T} & \delta u^{T}
\end{array}\right]\left[\begin{array}{ll}
H_{x x} & H_{x u} \\
H_{u x} & H_{u u}
\end{array}\right]\left[\begin{array}{l}
\delta x \\
\delta u
\end{array}\right] \\
& \bar{H}_{\delta u}=\bar{\lambda}^{T} F_{u}+\delta x^{T} H_{x u}+\delta u^{T} H_{u u}=0 \\
& \dot{\bar{\lambda}}=-\left(\bar{H}_{\delta x}\right)^{T}=-F_{x}^{T} \bar{\lambda}-H_{x x} \delta x-H_{x u} \delta u, \quad \bar{\lambda}\left(t_{f}\right)=\Phi_{x x} \delta x\left(t_{f}\right)
\end{aligned}
$$

Since the equations are linear, the NE solution $\delta u$ can be written explicitly. The key to the NE solution is the sweep method, where the adjoints are considered as linear functions of the states: $\bar{\lambda}=S \delta x$. From the dynamics 
of $\bar{\lambda}$ one can compute a differential equation for $S$ [3]. The explicit solution takes the following form:

$$
\begin{aligned}
\delta u & =-K \delta x \\
K & =H_{u u}^{-1}\left(H_{u x}+F_{u}^{T} S\right) \\
\dot{S} & =-H_{x x}-S F_{x}-F_{x}^{T} S+H_{x u} K+S F_{u} K \\
S\left(t_{f}\right) & =\Phi_{x x}\left(x\left(t_{f}\right)\right)
\end{aligned}
$$

The problem is non-singular if $H_{u u}$ is invertible, and singular otherwise. Thus, the feedback law (14)-(17) is only defined for non-singular problems.

\subsection{Link between NE approach and NCO-tracking}

As shown in Section 2.2, the neighboring extremal approach minimizes $\delta^{2} J$. In the following, it will be shown that it also forces the first-order variation of the NCO to zero, i.e. $\delta H_{u}=0$.

Theorem 1 The NE solution of the variational optimization problem (9)(10) forces the first-order variation of the NCO to zero, i.e. $\delta H_{u}=0$.

Proof: The first-order variation of the adjoints is given by

$$
\dot{\delta} \lambda=-F_{x}^{T} \delta \lambda-\delta F_{x}^{T} \lambda-\delta L_{x}^{T}, \quad \delta \lambda\left(t_{f}\right)=\Phi_{x x} \delta x\left(t_{f}\right)
$$

Using the fact that $\delta F_{x}=\sum_{k=1}^{n} \frac{\partial F_{x}}{\partial x_{k}} \delta x_{k}+\sum_{k=1}^{m} \frac{\partial F_{x}}{\partial u_{k}} \delta u_{k}$, noting that the same structure holds for $\delta L_{x}$, and regrouping to be able to introduce the Hamiltonian gives

$$
\dot{\delta} \lambda=-F_{x}^{T} \delta \lambda-H_{x x} \delta x-H_{x u} \delta u, \quad \delta \lambda\left(t_{f}\right)=\Phi_{x x} \delta x\left(t_{f}\right)
$$

Comparing (13) and (19), it can be seen that $\bar{\lambda}=\delta \lambda$.

The first-order variation of $H_{u}$ is given by

$$
\delta H_{u}=\delta\left(\lambda^{T} F_{u}\right)+\delta L_{u}=\delta \lambda^{T} F_{u}+\lambda^{T} \delta F_{u}+\delta L_{u}
$$

Using similar expressions for $\delta F_{u}$ and $\delta L_{u}$ gives:

$$
\delta H_{u}=\delta \lambda^{T} F_{u}+\delta u^{T} H_{u u}+\delta x^{T} H_{x u}
$$

A comparison of (12) and (21) gives $\bar{H}_{\delta u}=\delta H_{u}$. Thus, since the NE approach forces $\bar{H}_{\delta u}=0$, it also forces the first variation of the NCO to be zero, i.e. $\delta H_{u}=0$. 


\section{Neighboring Extremal Approach for Singu- lar Problems}

Singular situations, i.e. those with $H_{u u}=0$, are quite common. An important class of systems with that property is the class of input-affine systems with the integral cost being independent of the inputs. Here, $F(x, u)=$ $f(x)+g(x) u$ and $L_{u}=0$. So, $H_{u}=\lambda^{T} g(x)$ does not depend on the inputs, leading to $H_{u u}=0$. In such a case, the NE approach presented in the previous section cannot be used directly.

To compute the optimal inputs from $H_{u}=0$, the NCO needs to be differentiated with respect to time along the trajectories of the system until $u$ appears explicitly (the number of differentiations is termed the order of singularity). Similarly, to set up a NE controller, $\delta H_{u}$ will be differentiated with respect to time until $\delta u$ appears explicitly. This is where an interplay between the nonlinear dynamics and the linearized ones occurs as will be explained next. Since in the singular case, $H_{u u}=0, \delta H_{u}$ reads

$$
\delta H_{u}=\delta \lambda^{T} F_{u}+\delta x^{T} H_{x u}
$$

The time differentiation of $\delta H_{u}$ is given by

$$
\frac{d}{d t} \delta H_{u}=\dot{\delta \dot{\lambda}}{ }^{T} F_{u}+\delta \lambda^{T} \frac{d}{d t} F_{u}+\dot{\delta x}^{T} H_{x u}+\delta x^{T} \frac{d}{d t} H_{x u}
$$

Among the different terms $\dot{\delta x}$ and $\dot{\delta} \lambda$ ares obtained from the linearized dynamics, while the nonlinear dynamics is used for the time differentiations $\frac{d}{d t} F_{u}$ and $\frac{d}{d t} H_{x u}$, since the latter cannot be deduced from the linearization alone. Thus, it can be computed that

$$
\delta \dot{H}_{u}{ }^{T}=A_{1} \delta \lambda+B_{1} \delta x+C_{1} \delta u
$$

where $A_{1}=\dot{A}_{0}-A_{k-1} F_{x}^{T} B_{1}=\dot{B}_{0}+B_{0} F_{x}-A_{0} H_{x x}, C_{1}=B_{0} F_{u}-A_{0} H_{x u}$, with $A_{0}=F_{u}^{T}, B_{0}=H_{u x}$ and $C_{0}=H_{u u}$.

It will be shown next that the number of differentiations needed to make $\delta u$ appear in the differentiations of $\delta H_{u}$ is the same as the number of differentiations of $H_{u}$ needed to make $u$ appear. This is shown by proving the following:

$$
\frac{d^{k}}{d t^{k}} \delta H_{u}=\delta \frac{d^{k}}{d t^{k}} H_{u}
$$

Since $\forall k<\sigma, H_{u}$ is not a function of $u$, the proof will be simplified by considering a general function $G$ (representing in our case $H_{u}$ ) of $x$ only. 
Proposition 1 Consider the vector function $G(x)$, its first-order variation $\delta G$ and the time differentiations of $G$ along the dynamics $\dot{x}=F(x, u)$. Then, the following equality holds: $\delta \frac{d^{k}}{d t^{k}} G=\frac{d^{k}}{d t^{k}} \delta G \forall k$.

Proof: The proof proceeds by induction. For $k=1$,

$$
\begin{aligned}
\delta \frac{d}{d t} G & =\delta\left(G_{x} \dot{x}\right)=\delta\left(G_{x} F\right) \\
& =\sum_{j=1}^{n} \sum_{k=1}^{n} \delta x_{j} \frac{\partial^{2} G}{\partial x_{j} \partial x_{k}} F_{k}+G_{x} F_{x} \delta x+G_{x} F_{u} \delta u \\
\frac{d}{d t} \delta G & =\frac{d}{d t} G_{x} \delta x=\sum_{j=1}^{n} \sum_{k=1}^{n} \delta x_{j} \frac{\partial^{2} G}{\partial x_{j} \partial x_{k}} \dot{x}_{k}+G_{x} \dot{\delta x} \\
& =\sum_{j=1}^{n} \sum_{k=1}^{n} \delta x_{j} \frac{\partial^{2} G}{\partial x_{j} \partial x_{k}} F_{k}+G_{x} F_{x} \delta x+G_{x} F_{u} \delta u
\end{aligned}
$$

Simple inspection shows that equations (26) and (27) are identical. To continue the induction suppose, $\delta \frac{d^{k-1}}{d t^{k-1}} G=\frac{d^{k-1}}{d t^{k-1}} \delta G$. Then,

$$
\frac{d^{k}}{d t^{k}} \delta G=\frac{d}{d t} \frac{d^{k-1}}{d t^{k-1}} \delta G=\frac{d}{d t} \delta \frac{d^{k-1}}{d t^{k-1}} G=\delta \frac{d}{d t} \frac{d^{k-1}}{d t^{k-1}} G=\delta \frac{d^{k}}{d t^{k}} G
$$

follows.

The above result is fairly intuitive as time differentiation and computation of the first-order variation are linear operators and therefore the order of the operations can be interchanged.

\subsection{Computing the time differentiations}

From $(21), \delta H_{u}$ can be described as:

$$
\delta H_{u}^{T}=A_{0} \delta \lambda+B_{0} \delta x+C_{0} \delta u
$$

with $A_{0}=F_{u}^{T}, B_{0}=H_{u x}$ and $C_{0}=H_{u u}$. From (24), it can be seen that $\delta \dot{H}_{u}{ }^{T}$ inherits the structure of $\delta H_{u}$. Thus by induction, the first-order variation $\delta H_{u}$ and its time derivatives have the generic form

$$
\frac{d^{k}}{d t^{k}} \delta H_{u}^{T}=A_{k} \delta \lambda+B_{k} \delta x+C_{k} \delta u
$$


where the recursive law to describe the $k^{\text {th }}$ differentiation as a function of the $(k-1)^{\text {st }}$ differentiation can be written as

$$
\begin{aligned}
& A_{k}=\dot{A}_{k-1}-A_{k-1} F_{x}^{T} \\
& B_{k}=\dot{B}_{k-1}+B_{k-1} F_{x}-A_{k-1} H_{x x} \\
& C_{k}=B_{k-1} F_{u}-A_{k-1} H_{x u}
\end{aligned}
$$

The time differentiations stop at $k=\sigma$ when $C_{\sigma}$ becomes invertible. Then, as before the sweep method with $\delta \lambda=S \delta x$ can be used to compute the feedback law. It can be seen that the parts of the feedback law corresponding to (14), (16) and (17) remain unaltered, while the gain matrix (15) is replaced by

$$
K=C_{\sigma}^{-1}\left(A_{\sigma} S+B_{\sigma}\right)
$$

Thus, the first difference with non-singular problems is that, instead of inverting $C_{0}=H_{u u}$, the equations are differentiated with respect to time until $C_{\sigma}$ becomes invertible.

\subsection{Satisfaction of boundary conditions}

A second difference arises from the fact that, with the feedback gain (34), only $\frac{d^{\sigma}}{d t^{\sigma}} \delta H_{u}=0$ is enforced. Thus, this does not guarantee that the lower derivatives $\frac{d^{k}}{d t^{k}} \delta H_{u}$ are 0 for $k<\sigma$. One possibility to guarantee this would be to force all the $\sigma$ initial conditions to zero, i.e.

$$
\left[\frac{d^{k}}{d t^{k}} \delta H_{u}^{T}\right]_{t=0}=\left[A_{k} S+B_{k}\right]_{t=0} \delta x(0)=0 \quad \forall k<\sigma
$$

This way, the feedback law would ensure $\delta H_{u}=0$ for all $t$. However, in practice, this approach is not efficient since it is often not possible to enforce exactly the $\sigma$ initial conditions $\left(A_{k} S+B_{k}\right)_{t=0} \delta x(0)=0$.

Thus, since the handle for forcing $\delta H_{u}=0$ is only through its $\sigma^{\text {th }}$ derivative, fast asymptotically stable $\sigma^{t h}$-order dynamics are imposed on $\delta H_{u}$ so as to drive it quickly to zero:

$$
\frac{d^{\sigma}}{d t^{\sigma}} \delta H_{u}^{T}=-\sum_{k=0}^{\sigma-1} \gamma_{k} \frac{d^{k}}{d t^{k}} \delta H_{u}^{T}
$$

Replacing the terms in (36) by the expression found in (30) gives

$$
A_{\sigma} \delta \lambda+B_{\sigma} \delta x+C_{\sigma} \delta u=-\sum_{k=0}^{\sigma-1} \gamma_{k}\left(A_{k} \delta \lambda+B_{k} \delta x\right)
$$


This modifies only the $K$ matrix of the feedbak law (14)-(17):

$$
K=C_{\sigma}^{-1}\left[\left(A_{\sigma}+\sum_{k=0}^{\sigma-1} \gamma_{k} A_{k}\right) S+B_{\sigma}+\sum_{k=0}^{\sigma-1} \gamma_{k} B_{k}\right]
$$

The poles of $\delta H_{u}$ dynamics (determined by the choice of $\gamma_{k}$ ) have to be chosen carefully. On the one hand, fast poles lead to large corrections that might invalidate the linear approximation. Moreover, fast poles render the feedback highly sensitive to noise and may thus lead to poor results in terms of optimality. On the other hand, the poles have to be fast enough so that the perturbations are rejected sufficiently fast compared to the final time $t_{f}$.

\section{Illustrative example}

\subsection{Reaction system}

The reaction system $A+B \rightarrow C$ and $2 B \rightarrow D$ is considered in a semi-batch chemical reactor with the reactant $B$ being fed in, where $C$ is the desired product and $D$ an undesired side product [7]. The dynamics of the system can be described using the following equations:

$$
\begin{aligned}
\dot{c_{A}} & =-k_{1} c_{A} c_{B}-u \frac{c_{A}}{V}, & c_{A}(0) & =c_{A 0} \\
\dot{c_{B}} & =-k_{1} c_{A} c_{B}-2 k_{2} c_{B}^{2}-u \frac{\left(c_{B}-c_{B i n}\right)}{V}, & & c_{B}(0)=c_{B 0} \\
\dot{V} & =u, & & V(0)=V_{0}
\end{aligned}
$$

where $c_{A}$ and $c_{B}$ are the concentrations $\left(\frac{m o l}{l}\right)$ of the species $A$ and $B$, respectively, $V$ the volume $(l), k_{1}$ and $k_{2}$ the kinetic coefficients $\left(\frac{l}{m o l ~ h}\right), u$ the inlet feed rate $\left(\frac{l}{h}\right), c_{B i n}$ the inlet concentration $\left(\frac{m o l}{l}\right)$ and $c_{A 0}, c_{B 0}$, and $V_{0}$ the initial conditions. The initial conditions are: $c_{A 0}=0.72 \frac{\mathrm{mol}}{\mathrm{l}}, c_{B 0}=0.0614 \frac{\mathrm{mol}}{\mathrm{l}}$ and $V_{0}=1 l$. The numerical values of the parameters are: $k_{1}=0.053 \frac{l}{\text { mol } h}$, $k_{2}=0.128 \frac{\mathrm{l}}{\mathrm{mol} \mathrm{h}}, c_{\text {Bin }}=5 \frac{\mathrm{mol}}{\mathrm{l}}$.

\subsection{Optimization problem}

The optimization problem consists of maximizing $n_{C}\left(t_{f}\right)-n_{D}\left(t_{f}\right)$, i.e. the difference between the numbers of moles of $C$ and $D$ at final time. It can be 
shown that $n_{C}=c_{A 0} V_{0}-c_{A} V$ and $n_{D}=\frac{1}{2}\left(c_{B 0} V_{0}+c_{B i n}\left(V-V_{0}\right)-c_{B} V-c_{A 0} V_{0}+\right.$ $\left.c_{A} V\right)$. Removing the constant terms in $n_{C}\left(t_{f}\right)-n_{D}\left(t_{f}\right)$, the optimization problem can be formulated as the following minimization problem:

$$
\begin{aligned}
\min _{u(t)} J= & \left(3 c_{A}\left(t_{f}\right)+c_{B i n}-c_{B}\left(t_{f}\right)\right) V\left(t_{f}\right) \\
& \text { s.t. Dynamic equations }(39)-(41) \\
& 0 \leq u \leq u_{\max }
\end{aligned}
$$

The necessary conditions of optimality read:

$$
H_{u}=-\lambda_{1} \frac{c_{A}}{V}-\lambda_{2} \frac{\left(c_{B}-c_{B i n}\right)}{V}+\lambda_{3}=0
$$

Since $H_{u}$ is independent of $u$, the problem is singular. The time derivatives of $H_{u}$ take the form:

$$
\begin{aligned}
\dot{H}_{u}= & -\lambda_{1} \frac{k_{1} c_{A}\left(c_{B}-c_{B i n}\right)}{V}-\lambda_{2} \frac{k_{1} c_{A}\left(c_{B}-c_{B i n}\right)+2 k_{2} c_{B}\left(c_{B}-2 c_{B i n}\right)}{V}=0 \\
\ddot{H}_{u}= & \lambda_{1}\left(\frac{c_{B i n} k_{1} c_{A}\left(k_{1} c_{A}+4 k_{2} c_{B}\right)}{V}+2 \frac{u k_{1} c_{A}\left(c_{B}-c_{B i n}\right)}{V^{2}}\right) \\
& +\lambda_{2}\left(\frac{c_{B i n}\left(4 k_{1} c_{A} k_{2} c_{B}+8 k_{2}^{2} c_{B}^{2}+k_{1}^{2} c_{A}^{2}\right)}{V}+2 \frac{u\left(c_{B}-c_{B i n}\right)\left(k_{1} c_{A}+2 k_{2}\left(c_{B}-c_{B i n}\right)\right)}{V^{2}}\right)=0
\end{aligned}
$$

Since $u$ appears in the second time derivative of $H_{u}$, the order of singularity is $\sigma=2$. Eliminating the adjoint variables from (43)-(45), the singular input reads:

$$
u_{\text {sing }}=\frac{c_{B} V\left(k_{1} c_{A} c_{B}-2 k_{1} c_{A} c_{B i n}-4 k_{2} c_{B} c_{B i n}\right)}{2 c_{B i n}\left(c_{B}-c_{B i n}\right)}
$$

The initial conditions are chosen so that the optimal solution consists only of a singular arc. The optimal state and input trajectories arem shown in Figure 1.

\subsection{NE controller}

The computation of the NE controller starts with the (symbolic) computation of the matrix $A_{k}, B_{k}, C_{k}$ for $k=1,2$. For this purpose, equations (31)-(33) are used with $A_{0}$ and $B_{0}$ given by

$$
A_{0}=\left[\begin{array}{lll}
-\frac{c_{A}}{V} & -\frac{c_{B}-c_{B i n}}{V} & 1
\end{array}\right]
$$



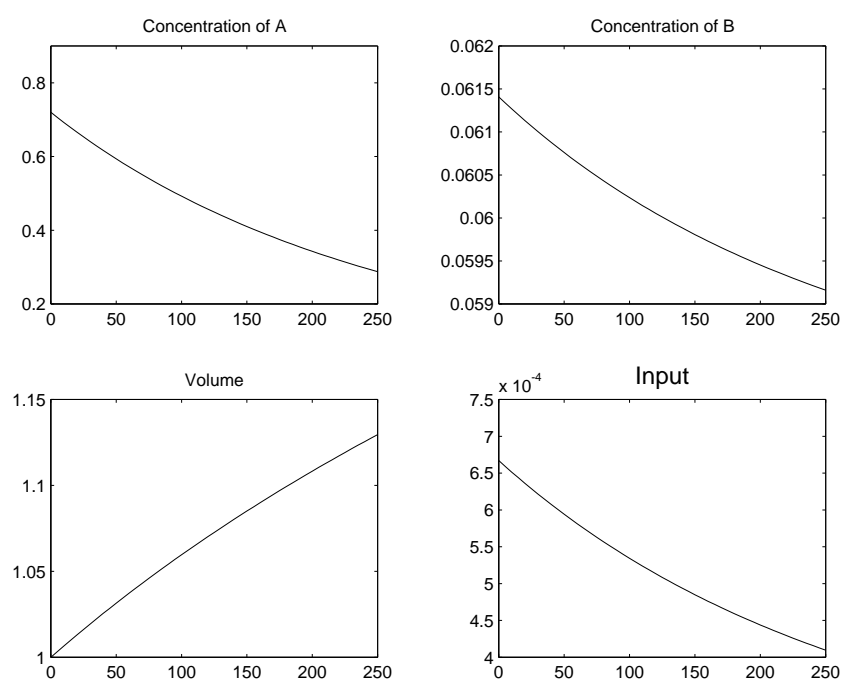

Figure 1: Optimal state and input trajectories.

$$
B_{0}=\left[\begin{array}{lll}
-\frac{\lambda_{1}}{V} & -\frac{\lambda_{2}}{V} & \frac{\lambda_{1} c_{A}+\lambda_{2}\left(c_{B}-c_{B i n}\right)}{V^{2}}
\end{array}\right]
$$

The expressions for $C_{k}$ are

$$
\begin{aligned}
& C_{0}=0, \quad C_{1}=0 \\
& C_{2}=2 \frac{\left(c_{B}-c_{B i n}\right)\left(\left(\lambda_{1}+\lambda_{2}\right) k_{1} c_{A}+2 \lambda_{2} k_{2}\left(c_{B}-c_{B i n}\right)\right)}{V^{2}}
\end{aligned}
$$

Note that, since $C_{2}$ is non-zero, its inverse can be used for calculating $K$ in (38). The numerical values are computed along the nominal solution using the corresponding adjoints. The values of $\gamma$ chosen to determine the dynamics of $H_{u}$ are: $\gamma_{0}=0.0016$ and $\gamma_{1}=0.1$, corresponding to two stable poles $(-0.02,-0.08)$.

\subsection{Simulation with perturbations}

In the simulations, a perturbation of $\pm 10 \%$ is introduced on the initial conditions. First, the nominal input is applied to the perturbed system. Then, the nominal input is used as a feedforward term with the NE controller providing the feedback term. The NE controller uses the numerical values of 


\begin{tabular}{|c|c|c|c|c|c|c|}
\hline$c_{A 0}$ & $c_{B 0}$ & $V_{0}$ & $J_{\text {opt }}$ & $J_{O L}$ & $J_{F B}$ & Recovery $(\%)$ \\
\hline 0.720 & 0.0614 & 1 & 6.5556 & 6.5556 & 6.5556 & - \\
0.648 & 0.0553 & 0.9 & 5.7801 & 5.7938 & 5.7802 & 99.78 \\
0.648 & 0.0675 & 0.9 & 5.7686 & 5.7843 & 5.7687 & 99.30 \\
0.792 & 0.0553 & 1.1 & 7.3685 & 7.3851 & 7.3686 & 99.27 \\
0.792 & 0.0675 & 1.1 & 7.3542 & 7.3684 & 7.3542 & 99.86 \\
\hline
\end{tabular}

Table 1: Results for different perturbation of the initial condition. $J_{\text {opt }}=$ optimal cost for the perturbed system, $J_{O L}=$ cost obtained by applying the nominal input open loop to the perturbed system, $J_{F B}=$ cost obtained by applying the nominal input along with the NE feedback to the perturbed system.

$K(t)$ and the nominal trajectories of the states. The following table displays the numerical results for four different simulations.

It can be seen from the table that the loss in optimality $\left(J_{O L}-J_{\text {opt }}\right)$ is small (less that $0.5 \%$ for $10 \%$ variation in all initial conditions) since the problem is singular, i.e. $H_{u u}=0$. So, only higher-order time derivatives contribute to the cost deviation. Furthermore, it can be seen that the proposed neighboring extremal feedback is able to almost completely recover the loss in optimality $\left(J_{F B} \approx J_{o p t}\right)$.

The simulation results for the initial conditions $c_{A 0}=0.792 \frac{\mathrm{mol}}{\mathrm{l}}, c_{B 0}=$ $0.0553 \frac{\mathrm{mol}}{\mathrm{l}}$, and $V_{0}=1.1 \mathrm{l}$ are depicted in Figure 2. It is clearly seen that the input and states of the perturbed system under NE feedback catch up quickly with the optimal trajectories of the perturbed system. The true optimum for the perturbed system consists of a short constraint-seeking arc, $u=u_{\max }$, so as to arrive at the sensitivity-seeking arc in minimum time. In the feedback solution, this arc is absent because the dynamics of $\delta H_{u}(36)$ are chosen rather slow. However, it can be seen that the loss of optimality due to the time necessary to catch up is only of the order of $0.01 \%$. Simulations with noise are not shown since the desired effects would be buried in noise.

\section{Conclusion}

The problem of tracking the conditions of optimality on the various arcs of an optimal solution has been addressed recently. Towards this end, the 

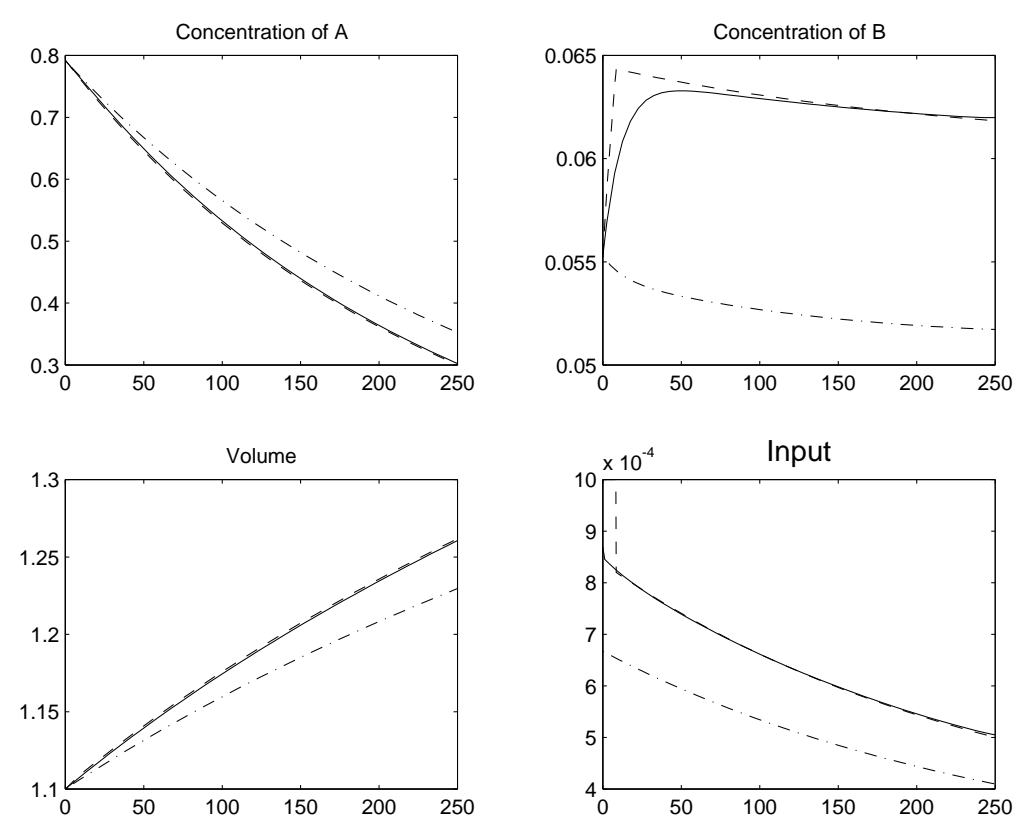

Figure 2: Simulation with perturbed initial conditions $\left(c_{A 0}=0.792 \frac{\mathrm{mol}}{\mathrm{l}}\right.$, $c_{B 0}=0.0553 \frac{\mathrm{mol}}{\mathrm{l}}$, and $V_{0}=1.1 \mathrm{l}$ ) (dashed line: optimal solution for the perturbed system; dotted-dashed line: perturbed system running open-loop with the nominal solution; solid line: system with NE feedback)

current paper revisits the neighboring extremal approach for the sensitivityseeking arcs. A formal connection between this approach and NCO-tracking is established. From this connection, the neighbouring extremal approach is extended to the singular problems. An example has been used to illustrate the application of the method.

Despite the possibly heavy symbolic and numerical computations required to compute the NE feedback, it should be noted that all this effort is done off-line, with the on-line calculation corresponding to simple state feedback. Thus, this approach can also be applied to fast dynamic system, independent of whether the system is singular or not.

\section{References}

[1] L.T. Biegler. Solution of dynamic optimization problems by successive quadratic programming and orthogonal collocation. Comp. Chem. Eng., 
8(5):243-248, 1984.

[2] A.E. Breakwell, J.L. Speyer, and A.E. Bryson. Optimization and control of nonlinear systems using the second variation. SIAM Journal on Control and Optimization, 1:193-217, 1963.

[3] A.E. Bryson and Y.C. Ho. Applied Optimal Control. Ginn and Company, 1969.

[4] A. Cruse, W. Marquardt, A. Helbig, and J.-S. Kussi. Optimizing adaptive calorimetric model predictive control of a benchmark semi-batch reaction process. IFAC World Congress, Barcelona, 2002, 2002.

[5] A.Y. Lee and L. Markus. Foundations of Optimal Control Theory. The SIAM series, 1967.

[6] B. Srinivasan, D. Bonvin, E. Visser, and S. Palanki. Dynamic optimization of batch processes - II: Role of measurements in handling uncertainty. Computers and Chemical Engineering, 27:27-44, 2003.

[7] B. Srinivasan, S. Palanki, and D. Bonvin. Dynamic optimization of batch processes - I: Characterization of the nominal solution. Computers and Chemical Engineering, 27:1-26, 2003. 